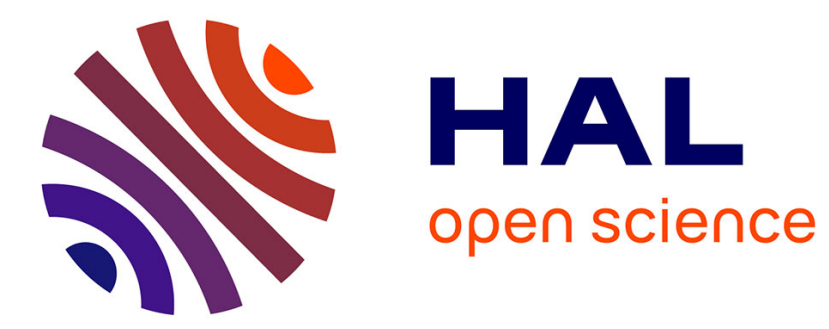

\title{
An exact method for graph coloring
}

\author{
Corinne Lucet, Florence Mendes, Aziz Moukrim
}

\section{To cite this version:}

Corinne Lucet, Florence Mendes, Aziz Moukrim. An exact method for graph coloring. Computers and Operations Research, 2006, 33 (8), pp.2189-2207. hal-00783637

\section{HAL Id: hal-00783637 \\ https://hal.science/hal-00783637}

Submitted on 4 Feb 2013

HAL is a multi-disciplinary open access archive for the deposit and dissemination of scientific research documents, whether they are published or not. The documents may come from teaching and research institutions in France or abroad, or from public or private research centers.
L'archive ouverte pluridisciplinaire HAL, est destinée au dépôt et à la diffusion de documents scientifiques de niveau recherche, publiés ou non, émanant des établissements d'enseignement et de recherche français ou étrangers, des laboratoires publics ou privés. 


\title{
An exact method for graph coloring*
}

\author{
C. Lucet, F. Mendes \\ LaRIA EA 2083, 5 rue du Moulin Neuf 80000 Amiens - France \\ (Corinne.Lucet, Florence.Mendes)@laria.u-picardie.fr \\ A. Moukrim \\ HeuDiaSyC UMR CNRS 6599 UTC, BP 2052960205 Compiègne - France \\ Aziz.Moukrim@hds.utc.fr
}

November 15, 2004

\begin{abstract}
We are interested in the graph coloring problem. We propose an exact method based on a linear-decomposition of the graph. The complexity of this method is exponential according to the linearwidth of the entry graph, but linear according to its number of vertices. We present some experiments performed on literature instances, among which COLOR02 library instances. Our method is usefull to solve more quickly than other exact algorithms instances with small linearwidth, such as mug graphs. Moreover, our algorithms are the first to our knowledge to solve the COLOR02 instance 4-Inser_3 with an exact method.
\end{abstract}

Keywords: graph coloring, exact method, linearwidth, linear-decomposition.

\section{Introduction}

The notions of tree-decomposition and path-decomposition have been introduced by Robertson and Seymour [23]. The decomposition method we propose here is strongly related to these notions, which have been studied in particular by Bodlaender to solve some NP-hard problems [1].

Our approach is a method based on successive decompositions of the representative graph providing successive resolved subgraphs and their corresponding

${ }^{*}$ with the financial support of Conseil Régional de Picardie and FSE 
separating sets named boundary sets. At each step, solutions of the resolved subgraph are represented by the different states of the boundary set vertices. The number of states to enumerate grows exponentially with the size of the boundary set. Its maximum size, for an optimal vertex numbering, corresponds to the linearwidth of the graph. The main advantage of this method is that the exponential factor of its complexity does not depend on the size of the graph but only on its linearwidth. This technique has been implemented efficiently by Carlier, Lucet and Manouvrier to solve various NP-hard problems such as network reliability or minimal Steiner tree computation $[4,18,19]$.

We apply the decomposition method to one of the most studied problems of combinatorial optimization: the graph coloring problem. It constitutes a central problem in a lot of applications such as school timetabling, scheduling, or frequency assignment $[5,6]$. The graph coloring problem consists in coloring the vertices of a graph with a minimum number of colors, ensuring that two adjacent vertices do not receive the same color. Various heuristic approaches have been proposed for this NP-hard problem [12]: greedy algorithms such as DSATUR [3], metaheuristics based on local search, tabu method, simulated annealing, hybrid algorithms, etc. (see for example $[10,11,13,16,20,22,26]$ ). To our knowledge, few exact methods are proposed to resolve this problem. One of the most well-known exact algorithms is the exact branch-and-bound algorithm implemented by Brelaz that uses DSATUR principles [3]. Implicit enumeration strategies are used in [17, 25, 27]. Mehrotra and Trick [21] studied a linear programming formulation which is solved by using column generation techniques. More recently, Mendez Diaz and Zabala presented a branch-and-cut algorithm $[8,9]$. Herrmann and Hertz presented efficient algorithms used to find edge-critical and vertex-critical subgraphs that have same chromatic numbers as initial graphs but are easier to solve [15]. Desrosiers, Galinier and Hertz proposed different algorithms to detect these critical subgraphs [7]. They made experiments on random graphs and on different types of benchmark graphs. Their method is very efficient on several instances families.

Our paper is organized as follows. In section 2, we describe the decomposition method and introduce the necessary notions. In section 3, we develop the implementation of the method. We present an exact algorithm which enables us to solve efficiently large instances whose linearwidth is bounded. Computational results obtained on various instances are presented in section 4 . They compare with two exact methods: a branch-and-cut algorithm [9] and an algorithm based on vertex-critical subgraphs detection [7]. Finally, we give some conclusions and discuss about the perspectives of this work. 


\section{Graph decompositions}

To introduce the kind of decomposition that we use to solve the graph coloring problem, it is necessary to recall some graph theory definitions and the notions of tree-decomposition and path-decomposition.

\subsection{Preliminary definitions}

An undirected graph $G$ is a pair, $G=(V, E)$, made up of a vertex set $V$ and an edge set $E \subset V \times V$. A graph $G$ is connected if for all vertices $w, v \in V(w \neq v)$, there exists a path from $w$ to $v$. Without loss of generality, the graphs $G$ we will consider in the following of this paper will be undirected and connected graphs. A subgraph of $G=(V, E)$, induced by $W \subseteq V$, is a graph $G(W)=\left(W, E_{W}\right)$ such that $E_{W}=E \cap(W \times W)$. A tree is a simple undirected graph, $T=\left(I, E_{T}\right)$, without cycle and with $\left|E_{T}\right|=|I|-1$. A rooted tree is a tree directed from the root $r$ to the leaves. If the edge $(p, v)$ belongs to a rooted tree, $p$ is the father of $v$, and $v$ is one of the sons of $p$.

\subsection{Tree-decomposition}

A tree-decomposition of $G=(V, E)$ is a pair $\left(\left\{X_{i} / i \in I\right\}, T=\left(I, E_{T}\right)\right)$ with $\left\{X_{i} / i \in I\right\}$ a family of subsets of $V$ and $T$ a tree such that:

$-\bigcup_{i \in I} X_{i}=V$,

- for all edges $(v, w) \in E$, there exists a subset $X_{i}, i \in I$, with $v \in X_{i}$ and $w \in X_{i}$,

- for all $i, j, k \in I$, if $j$ is on the path from $i$ to $k$ in $T$ then $X_{i} \cap X_{k} \subseteq X_{j}$. The treewidth of a tree-decomposition is $\max _{i \in I}\left(\left|X_{i}\right|-1\right)$. The treewidth of a graph $G$ is the minimum treewidth over all possible tree-decompositions of $G$.

The decomposition method is as follows. Given a tree-decomposition of the graph, partial solutions are built on the subsets $X_{i}$ and then associated to solve the considered problem. The decomposition method computes the solutions from the leaves to the root of the tree $T$, by examinating all partial solutions on every subgraph $G\left(X_{i}\right)$. The number of partial solutions is exponential according to the size of the subgraphs $X_{i}$. These partial solutions are computed from the solutions of $X_{f}$, for all $f$ belonging to the sons of $i$ in $T$. Unlike a simple enumerative method, this method allows one to factorize partial solutions of the $X_{i}$ sets into classes. This factorization provides an efficient method if the cardinality of the sets $X_{i}$ is small, i.e. if the treewidth of the tree-decomposition is sufficiently small. 
Whereas for some graph families, such as trees and serie-parallel graphs, one can compute the treewidth in linear time, computing the treewidth of any graph is a NP-complete problem [24]. Bodlaender [2] gives for a constant $k$ an algorithm in $O(n)$ which for a graph $G$ solves the problem "is the treewidth of $G$ at most $k$ ?". If so, it determines a tree-decomposition with treewidth at most $k$. This algorithm based on clique search and graph contraction has an exponential complexity with respect to $k\left(O\left(n * 2^{k^{2}}\right)\right)$. It cannot be used in practice, even for $k=4$.

\subsection{Path-decomposition and linear-decomposition}

The decomposition method that we will use in the following is based on a special case of tree-decomposition. We will consider a tree with only one leaf, that is a path.

A path-decomposition $\left(X_{1}, \ldots, X_{r}\right)$ of a graph $G$ is an ordered sequence of subsets of $V$ such that:

- $\bigcup_{1 \leq i \leq r} X_{i}=V$,

- for all edges $(v, w) \in E$, there exists a subset $X_{i}, 1 \leq i \leq r$, with $v \in X_{i}$ and $w \in X_{i}$,

- for all $i, j, k \in\{1, \ldots, r\}$, if $i \leq j \leq k$ then $X_{i} \cap X_{k} \subseteq X_{j}$.

The pathwidth of a path-decomposition is $\max _{1 \leq i \leq r}\left(\left|X_{i}\right|-1\right)$. The pathwidth of a graph is the minimum pathwidth over all possible path-decompositions of $G$. A vertex linear ordering of $G$ is a bijection $\mathcal{N}: V \rightarrow\{1, \ldots,|V|\}$. For more clarity, we denote $k$ the vertex $\mathcal{N}^{-1}(k)$. Let $F_{i}=\{j \in V / \exists(j, l) \in$ $E j \leq i<l\} \forall i \in\{1, \ldots,|V|\}$. The linearwidth of a vertex linear ordering $\mathcal{N}$ is $F_{\text {max }}(\mathcal{N})=\max _{i \in V}\left(\left|F_{i}\right|\right)$. The linearwidth of $G$, written $F_{\text {max }}(G)$, is the minimum linearwidth over all possible vertex linear orderings of $G$. The linearwidth of a graph equals its pathwidth [19].

Computing the pathwidth or the linearwidth of any graph is a NP-complete problem [24], similarly as computing the treewidth of any graph. The treewidth of a graph $G$ is smaller or equal to its pathwidth, and as a consequence the exponential factor of a tree-decomposition is smaller than that of a path-decomposition. Nevertheless, implementing the decomposition method on a lineardecomposition is easier than using a tree-decomposition. First, from a technical point of view, several $X_{i}$ partial solutions may have to be stored in memory when resolving a problem with a tree-decomposition. It involves some problems of memory storage and combination of the $X_{i}$ when implementing the algorithm. Moreover, creating a linear-decomposition is easier than creating a 
tree-decomposition. Thus, we use a vertex linear ordering of the graph to resolve the graph coloring problem with a linear-decomposition. The resolution method is then based on a sequential insertion of the vertices, using a vertex linear ordering previously determined. This will be developed in the following section.

\section{Application to the graph coloring problem}

In this section, we propose a method which uses linear-decomposition in order to solve the graph coloring problem.

\subsection{Problem definition}

A coloring of a graph $G=(V, E)$ is an assignment of a color $c(i) \in I$ to each vertex such that $c(i) \neq c(j)$ for all edges $(i, j) \in E$.

If the cardinality of $I$ is $k$, the coloring of $G$ is called a $k$-coloring. The minimum value of $k$ for which a k-coloring is possible is called the chromatic number of $G$ and is denoted $\chi(G)$. The graph coloring problem consists in finding the chromatic number of a graph.

\subsection{Linear decomposition principle}

Consider a graph $G=(V, E)$. Let $N=|V|$ and $M=|E|$. The vertices of $G$ are numbered according to a linear ordering $\mathcal{N}: V \rightarrow\{1, \ldots, N\}$. Let $V_{i}$ be subset of $V$, made of the vertices numbered from 1 to $i$. Let $H_{i}=\left(V_{i}, E_{i}\right)$ be the subgraph of $G$ induced by $V_{i}$. $F_{i}$ is the boundary set of $H_{i}$, i.e. the subset of $V_{i}$ such that $v \in F_{i}$ if and only if $\exists(v, w) \in E$ and $v \leq i<w$ (see figure 1). Let $H_{i}^{\prime}=\left(V_{i}^{\prime}, E_{i}^{\prime}\right)$ be the subgraph of $G$ induced by $V_{i}^{\prime}=\left(V \backslash V_{i}\right) \cup F_{i}$. Any kind of relation between the vertices of $H_{i}$ and those of $H_{i}^{\prime}$ depends on the vertices of $F_{i}$.

The linear decomposition is a dynamic method. During the coloring we will consider $N$ subgraphs $H_{1}, \ldots, H_{N}$ and the $N$ corresponding boundary sets $F_{1}, \ldots, F_{N}$. Starting from a vertex linear ordering, we build at first iteration a subgraph $H_{1}$ which contains only the vertex 1 , then at each step the next vertex and its corresponding edges are added, until $H_{N}$. Partial solutions of step $i$ are built from partial solutions of step $i-1$.

At each subgraph $H_{i}$ corresponds a boundary set $F_{i}$ containing the vertices of $H_{i}$ which have at least one neighbor in $H_{i}^{\prime}$. The boundary set $F_{i}$ is built from $F_{i-1}$ by adding the vertex $i$ and removing the vertices that have no neighbor with 

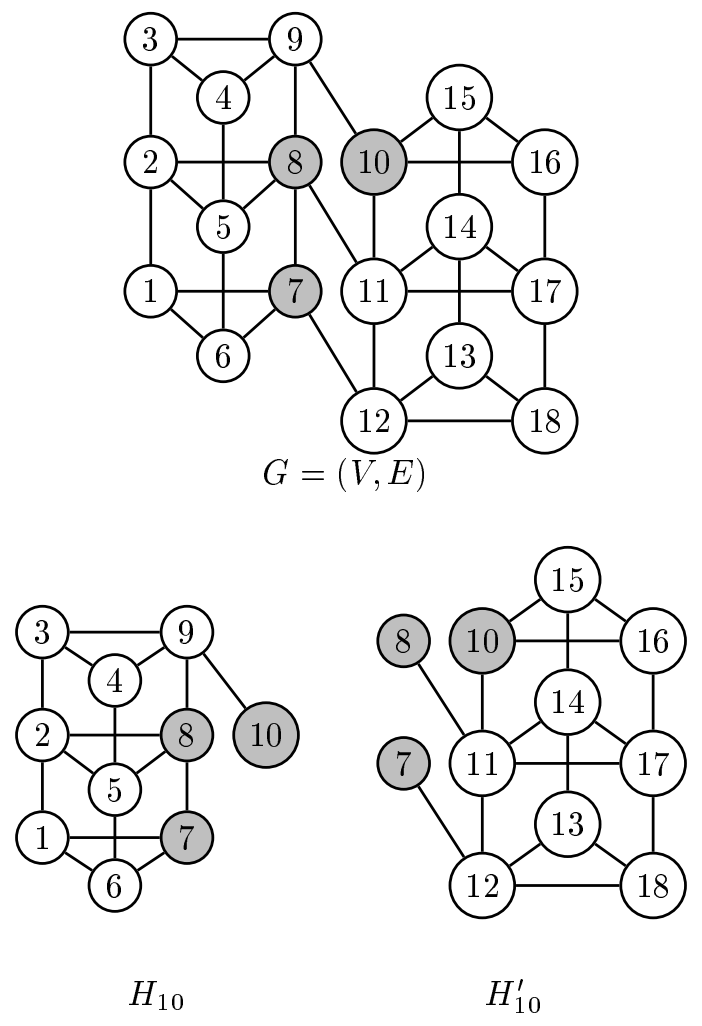

Figure 1: Subgraph $H_{10}$ of $G$ and its boundary set $F_{10}=\{7,8,10\}$ 


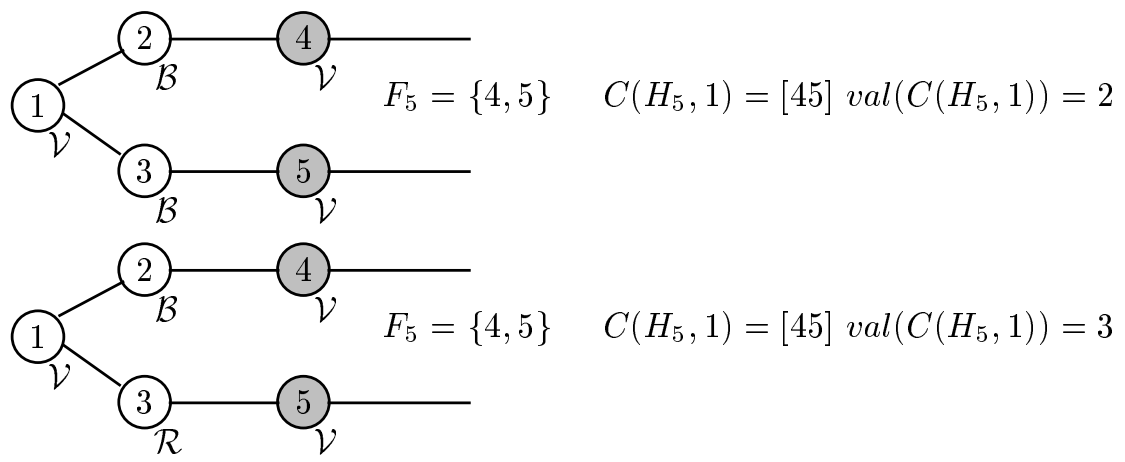

Figure 2: Different colorings of $H_{5}$ but same configuration of $F_{5}$

an ordering number greater than $i$. Several colorings of $H_{i}$ may correspond to the same coloring of $F_{i}$ (see figure 2). Moreover, the colors used by the vertices $V_{i} \backslash F_{i}$ do not interfere with the coloring of the vertices which have an ordering number greater than $i$, since no edge exists between them. So, only the partial solutions corresponding to different colorings of $F_{i}$ have to be stored in memory. This way, several partial solutions on $H_{i}$ may be summarized by a unique partial solution on $F_{i}$, called configuration of $F_{i}$.

The graph coloring problem is solved by evaluating at each step the configurations of the boundary set $F_{i}$. At step $i$, the subgraph $H_{i-1}$ is solved. It means that to each configuration of $F_{i-1}$ corresponds a value of the minimum number of colors necessary to color $H_{i-1}$ for this fixed coloring of the boundary set vertices. Then, partial solutions are built using solutions of the precedent step. This point will be detailed in section 3.4.

\subsection{Boundary set configurations}

A configuration of the boundary set $F_{i}$ is a given coloring of the vertices of $F_{i}$. This can be represented by a partition of $F_{i}$, denoted $B_{1}, \ldots, B_{j}$, such that two vertices $u, v$ of $F_{i}$ are in the same block $B_{c}$ if and only if they have the same color. The number of configurations of $F_{i}$ depends obviously on the number of edges between the vertices of $F_{i}$. The minimum number of configurations is 1 . If the vertices of $F_{i}$ form a clique, only one configuration is possible: $B_{1}, \ldots, B_{\left|F_{i}\right|}$, with exactly one vertex in each block. The maximal number of configurations of $F_{i}$ equals the number of partitions of a set with $\left|F_{i}\right|$ elements. When no edge exists between the boundary set vertices, all the partitions are to be considered.

The number of partitions of a set composed by $i$ elements and $j$ blocks, 
Table 1: Classification of the partitions of sets containing from 1 to 4 elements

\begin{tabular}{|l||l|l|l|l|}
\hline $\mathcal{A}_{i, j}$ & $\mathrm{j}=1$ & $\mathrm{j}=2$ & $\mathrm{j}=3$ & $\mathrm{j}=4$ \\
\hline \hline $\mathrm{i}=1$ & $\mathbf{1}[1]$ & & & \\
\hline $\mathrm{i}=2$ & $\mathbf{1}[12]$ & $\mathbf{2}[1][2]$ & & \\
\hline $\mathrm{i}=3$ & $\mathbf{1}[123]$ & $\mathbf{2}[13][2]$ & $\mathbf{5}[1][2][3]$ & \\
& & $\mathbf{3}[1][23]$ & & \\
& & $\mathbf{4}[12][3]$ & & \\
\hline $\mathrm{i}=4$ & $\mathbf{1}[1234]$ & $\mathbf{2}[134][2]$ & $\mathbf{9}[14][2][3]$ & $\mathbf{1 5}[1][2][3][4]$ \\
& & $\mathbf{3}[13][24]$ & $\mathbf{1 0}[1][24][3]$ & \\
& & $\mathbf{4}[14][23]$ & $\mathbf{1 1}[1][2][34]$ & \\
& & $\mathbf{5}[1][234]$ & $\mathbf{1 2}[13][2][4]$ & \\
& & $\mathbf{6}[124][3]$ & $\mathbf{1 3}[1][23][4]$ & \\
& & $\mathbf{7}[12][34]$ & $\mathbf{1 4}[12][3][4]$ & \\
& & $\mathbf{8}[123][4]$ & & \\
\hline
\end{tabular}

written $A_{i, j}$, is given by the recursive formula of Stirling numbers of the second kind:

$$
A_{i, j}=j \cdot A_{i-1, j}+A_{i-1, j-1}
$$

with $A_{1,1}=1$ and $A_{i, j}=0$ if $i<j$.

The number $T\left(F_{i}\right)$ of different partitions of the boundary set $F_{i}$ equals the sum of the $A_{\left|F_{i}\right|, j}$ for $j$ from 1 to $\left|F_{i}\right|$.

$$
T\left(F_{i}\right)=\sum_{j=1 \text { to }\left|F_{i}\right|} A_{\left|F_{i}\right|, j}
$$

To identify the configurations of the boundary set, we associate to each one an ordering number between 1 and $T\left(F_{i}\right)$. The partitions of sets with at most four elements and their ordering number are reported in table 1.

Let $C\left(H_{i}, x\right)$ be the $x^{t h}$ configuration of $F_{i}$ for the subgraph $H_{i}$. Its value, denoted $\operatorname{val}\left(C\left(H_{i}, x\right)\right)$, equals the minimum number of colors necessary to color $H_{i}$ for this configuration.

In figure 2, two different colorings of $H_{5}$ correspond to the same configuration of $F_{5}$. Only 2 colors are necessary to color $H_{5}$ with the configuration for which vertices 2 and 3 have a same color, whereas 3 colors are necessary when vertices 2 and 3 have different colors. The value of the configuration $C\left(H_{5}, 1\right)$, represented by the partition [45], is 2 , because we keep only the best valuation. 


\subsection{Coloring algorithm}

The details of the implementation of the decomposition method are reported in algorithm 1. Note that $H_{1}=(\{1\}, \emptyset)$ and $F_{1}=\{1\}$. So, there is only one configuration of $F_{1}, C\left(H_{1}, 1\right)=[1]$. The insertion of the vertex 2 of $G$ in $C\left(H_{1}, 1\right)$ can provide one or two configurations of $F_{2}$ (only one configuration if the vertices 1 and 2 are neighbors, two configurations otherwise).

At step $i$, we do not examine all the possible configurations of the step $i-1$, but only those which have been created at precedent step, it means those for which there is no edge between two vertices of the same block. For each configuration of $F_{i-1}$, we introduce the vertex $i$ in each block successively. Each time the introduction is possible without breaking the coloring rules, the corresponding configuration of $F_{i}$ is generated. We generate also the configurations obtained by adding to each configuration of $F_{i-1}$ a new block containing the vertex $i$.

For a given subgraph $H_{i}$, only the configurations that are different are represented. Their ordering number $x$, included between 1 and $T\left(F_{i}\right)$, is computed by an algorithm according to their number of blocks and their number of elements. When different colorings of $H_{i}$ correspond to the same configuration of $F_{i}$, only the best valuation is kept in $\operatorname{val}\left(C\left(H_{i}, x\right)\right)$.

At step $N$, only one configuration $C\left(H_{N}, 1\right)$ is generated from configurations of step $N-1$. It represents all the optimal coloring solutions and its value equals $\chi(G)$.

\section{Example of configuration computing.}

Assume that we are searching for a coloring of the graph $G$ of figure 3 and that we are at step $i$ with $F_{i}=\{u, v, w, i\}$.

Suppose that at step $i-1$, we had $F_{i-1}=\{u, v, w\}$ and that the configurations of $F_{i-1}$ were:

- $C\left(H_{i-1}, 2\right)=[u w][v]$ with value $\alpha$.

- $C\left(H_{i-1}, 4\right)=[u v][w]$ with value $\beta$.

- $C\left(H_{i-1}, 5\right)=[u][v][w]$ with value $\gamma$.

The values of $\alpha$ and $\beta$ are at least 2, since the corresponding configurations have 2 blocks. Remark that these values may be upper than 2 , depending on the configurations of the preceeding steps. By the same way, $\gamma$ is at least 3 .

We want to generate the configurations of $F_{i}$ from the configurations of $F_{i-1}$.

- it is impossible to insert $i$ in the first block of $C\left(H_{i-1}, 2\right)$, since $u$ and $i$ are neighbors. It is possible to insert $i$ in the second block of $C\left(H_{i-1}, 2\right)$. We obtain $C\left(H_{i}, 3\right)=[u w][v i]$ and $\operatorname{val}\left(C\left(H_{i}, 3\right)\right)=\alpha$. It is also possible to 


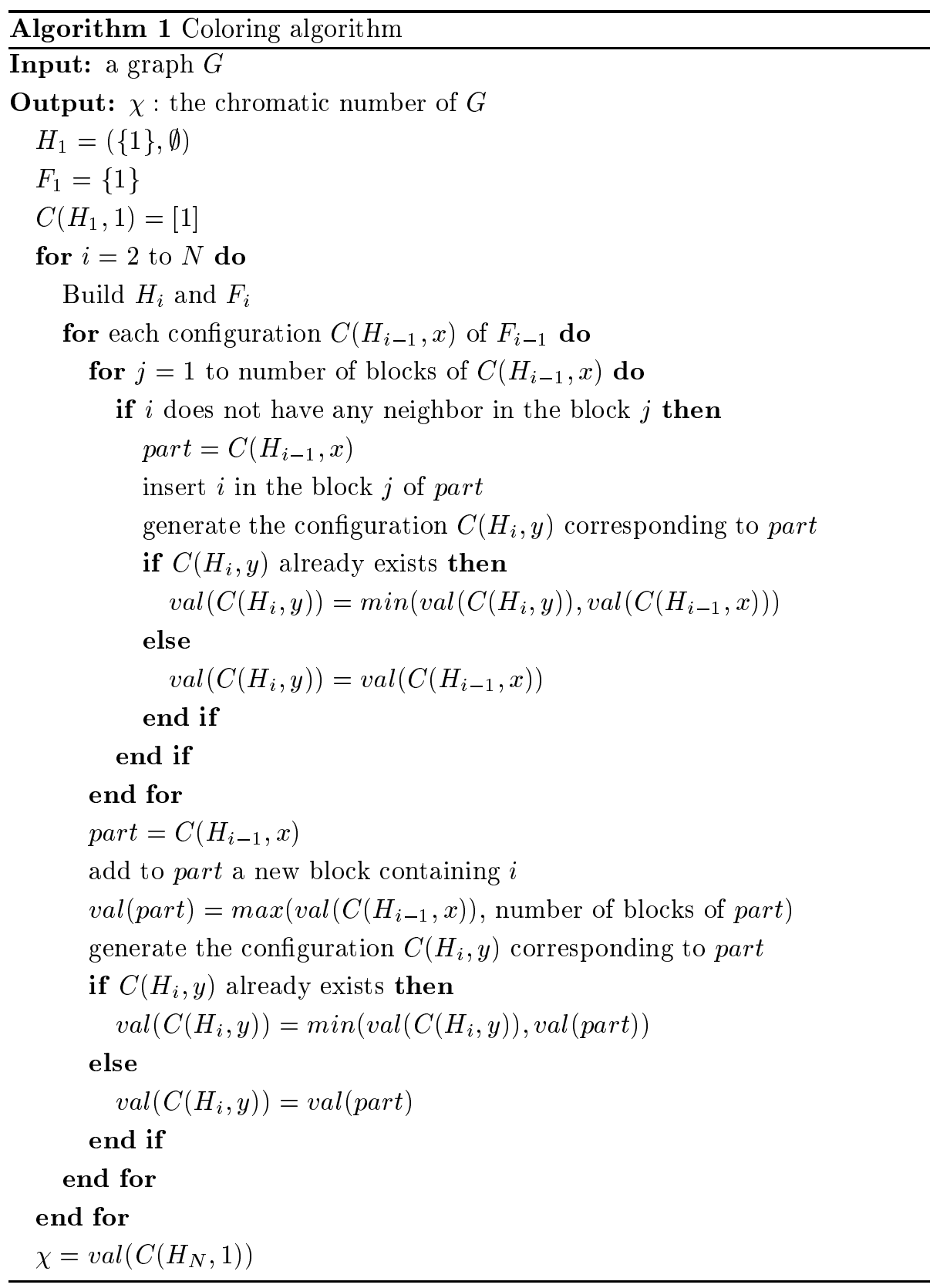




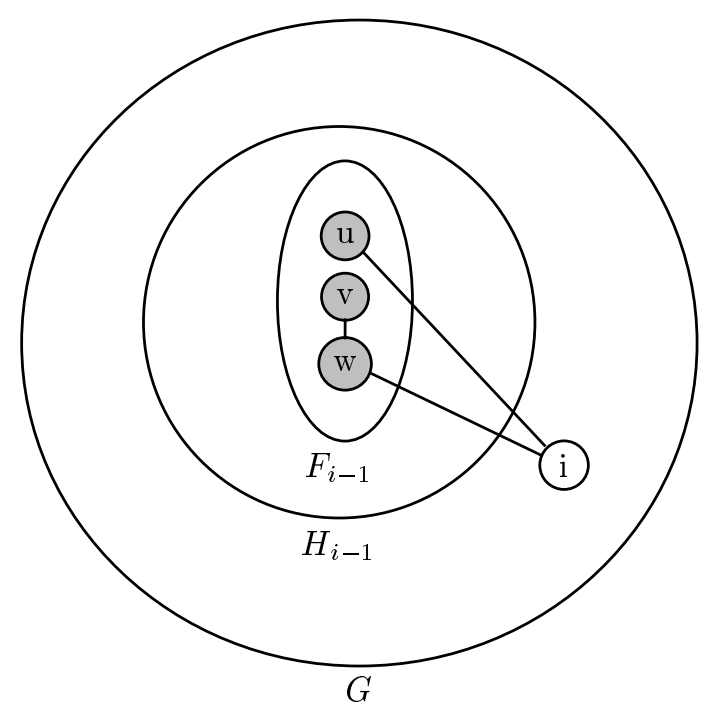

Figure 3: Construction of $H_{i}=\left(V_{i-1} \cup\{i\}, E_{i-1} \cup\{(u, i),(w, i)\}\right)$

add a third block, it provides the configuration $C\left(H_{i}, 12\right)=[u w][v][i]$ with $\operatorname{val} C\left(H_{i}, 12\right)=\max (\alpha, 3)$, since for this configuration at least three colors are needed.

- we must add a block to introduce $i$ in the configuration $C\left(H_{i-1}, 4\right)$. We obtain $C\left(H_{i}, 14\right)=[u v][w][i]$ with value $\max (\beta, 3)$.

- $i$ may be introduced in the second block of $C\left(H_{i-1}, 5\right)$. It provides the configuration $C\left(H_{i}, 10\right)=[u][v i][w]$ with value $\gamma$. By adding a new block, the configuration $C\left(H_{i}, 15\right)$ is created with $\operatorname{val}\left(C\left(H_{i}, 15\right)\right)=\max (\gamma, 4)$.

Thus five configurations are provided at step $i$, they are used to determine the configurations of the following step, and so on until the whole graph is colored.

\subsection{Improved coloring algorithm}

The number of configurations of a boundary set $F_{i}$ is exponential according to $\left|F_{i}\right|$. Moreover, a coloring represented by a configuration of $k$ blocks needs at least $k$ colors. By applying a succession of k-colorings (see algorithm 2), we avoid examining the configurations of more than $k$ blocks, which proves to be very interesting when $F_{\max }$ is larger than $\chi(G)$.

Take again the example of figure 3, and suppose that we are now searching for a 3-coloring of the graph $G$. All the configurations are made of at most three blocks, so their values are upper bounded by 3 . This time, only four 


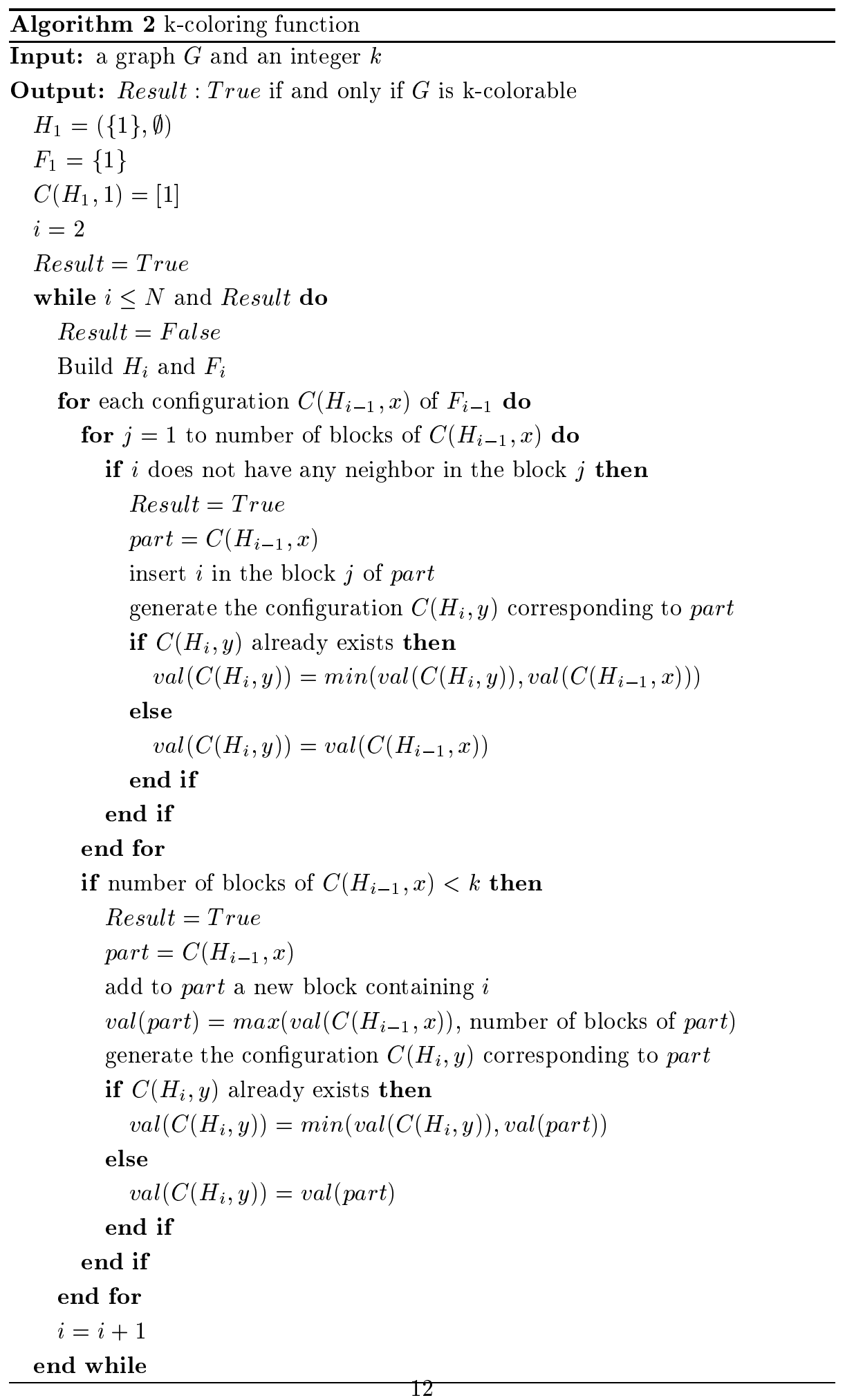




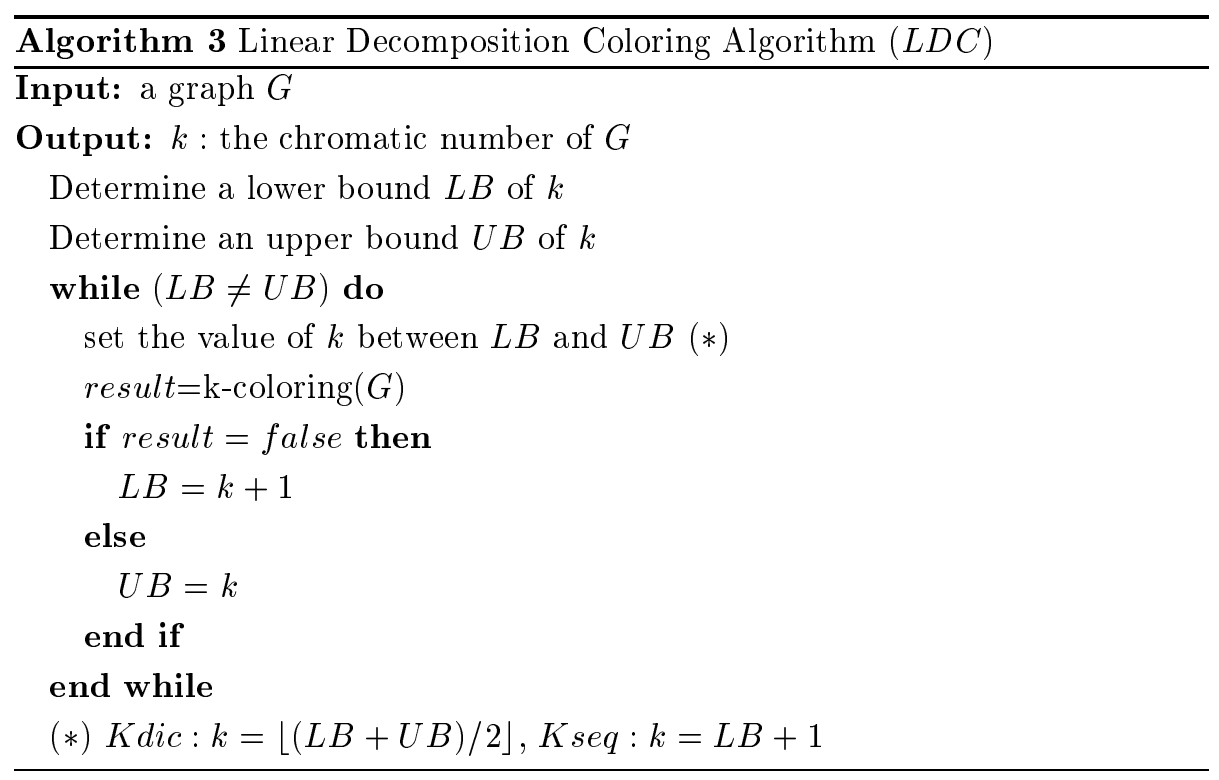

configurations are provided at step $i$ :

$C\left(H_{i}, 3\right)=[u w][v i]$ with value 2 or $3, C\left(H_{i}, 12\right)=[u w][v][i]$ with value 3 ,

$C\left(H_{i}, 14\right)=[u v][w][i]$ with value 3 , and $C\left(H_{i}, 10\right)=[u][v i][w]$ with value 3 .

We cannot add a new block to $C\left(H_{i-1}, 5\right)$, because it has already got three blocks.

The gain may obviously be more significant when the size of the boundary set increases. Indeed, let $T^{\prime}\left(F_{i}\right)$ be the maximum number of partitions of $F_{i}$ which have at most $k$ blocks:

$$
T^{\prime}\left(F_{i}\right)=\sum_{j=1 \text { to } k} A_{\left|F_{i}\right|, j}
$$

When no edge exists between the vertices of $F_{i}$, the gain $\operatorname{Gain}\left(F_{i}\right)$ for this step equals $T\left(F_{i}\right)$ minus $T^{\prime}\left(F_{i}\right)$ :

$$
\operatorname{Gain}\left(F_{i}\right)=\sum_{j=k+1 \text { to }\left|F_{i}\right|} A_{\left|F_{i}\right|, j}
$$

In the improved Linear Decomposition Coloring algorithm $(L D C)$, we start by determining a lower bound $L B$ of the chromatic number of $G$ (see algorithm 3). For that, we apply on $G$ some heuristics based on triangulated graphs. Indeed, it is easy to compute the chromatic number of a triangulated graph [14]. The used lower bound functions as follows: as long as the graph is not triangulated, we remove a vertex of smallest degree, and then we color the remaining triangulated graph by determining a perfect elimination order [14] on the vertices 
of $G$. The upper bound $U B$ is obtained by applying the heuristic DSATUR [3]. Then we apply a succession of k-colorings, $k$ varying between $L B$ and $U B$. We consider two different versions of $L D C$, depending on the way the value of $k$ is set: by dichotomy, denoted $K$ dic- $L D C$, or sequentially growing from $L B$ to $U B$, denoted $K$ seq- $L D C$. In theory, $K$ dic- $L D C$ has a better complexity than $K s e q-L D C$. Nevertheless, in practice $K s e q-L D C$ gives good lower bounds of the chromatic number of hard instances that $K d i c-L D C$ is unable to solve.

Algorithm 3 does not produce directly a coloring, nevertheless it can be obtained easily. All the configurations generated during the k-coloring have to be stored in memory. The color 1 is assigned to vertex $N$. When a k-coloring is found, a configuration of $F_{N-1}$ corresponding to the configuration of $F_{N}$ is chosen, a color is assigned to vertex $N-1$, and so on until vertex 1 is colored. Assign colors to the vertices needs a lot of memory space but does not increase the time complexity of the algorithm.

\subsection{The vertex linear ordering}

The maximum size of the boundary sets $F_{\max }(\mathcal{N})$ depends on the vertices numbering chosen (see figure 4). It corresponds to the linearwidth of the vertex linear ordering used to build the different subgraphs $H_{i}$ (cf section 2.3).

The complexity of the linear-decomposition is exponential with respect to $F_{\max }(\mathcal{N})$, so it is necessary to make a good choice when numbering the vertices of the graph. Unfortunately, finding an optimal vertex linear ordering in order to obtain the smallest linearwidth is a NP-complete problem [1]. A first method to reduce the size of the boundary sets is to number the vertices by applying a double BFS [14] on the graph. It produces the numbering $\mathcal{N}_{1}$. An other method is to begin the numbering from a clique and then order the vertices by decreasing number of edges with already numbered vertices. It produces the numbering $\mathcal{N}_{2}$. The aim of this method is to give the smallest numbers as possible to the vertices of boundary sets of size $F_{\max }\left(\mathcal{N}_{2}\right)$. To compare the linearwidths of these numberings with the linearwidth of the initial vertex numbering $\mathcal{N}_{0}$, we performed tests on random graphs $G_{N, d}$ (graphs with $N$ vertices and with density $d$ ) and on COLOR02 computational symposium instances. Some representative results are reported in Table 2. For random graphs, results over average of 5 instances are given.

The numbering $\mathcal{N}_{2}$ gave smaller $F_{\max }(\mathcal{N})$ for more instances than the other numberings, so we use this ordering in experiments of section 4 . Despite this, the number of configurations increases sometimes very strongly when introducing a 

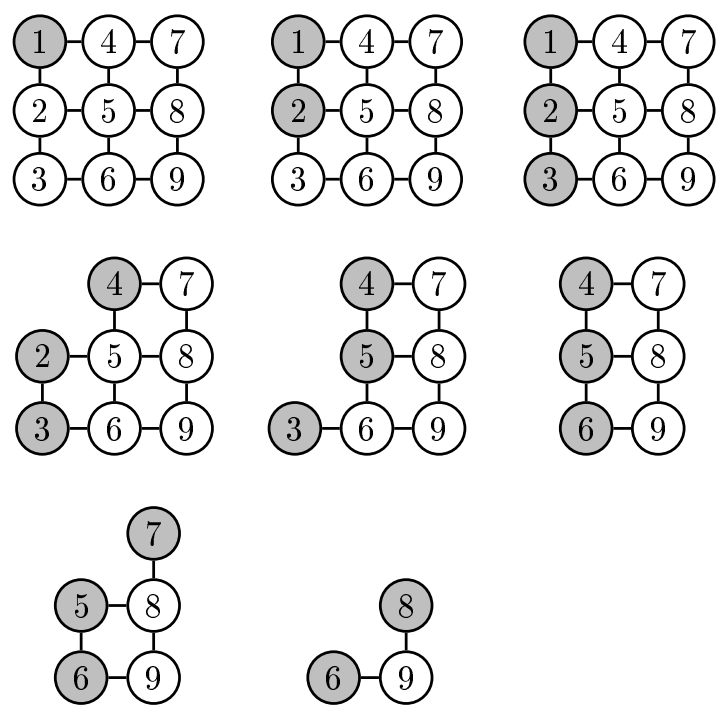

$F_{\text {max }}=3$
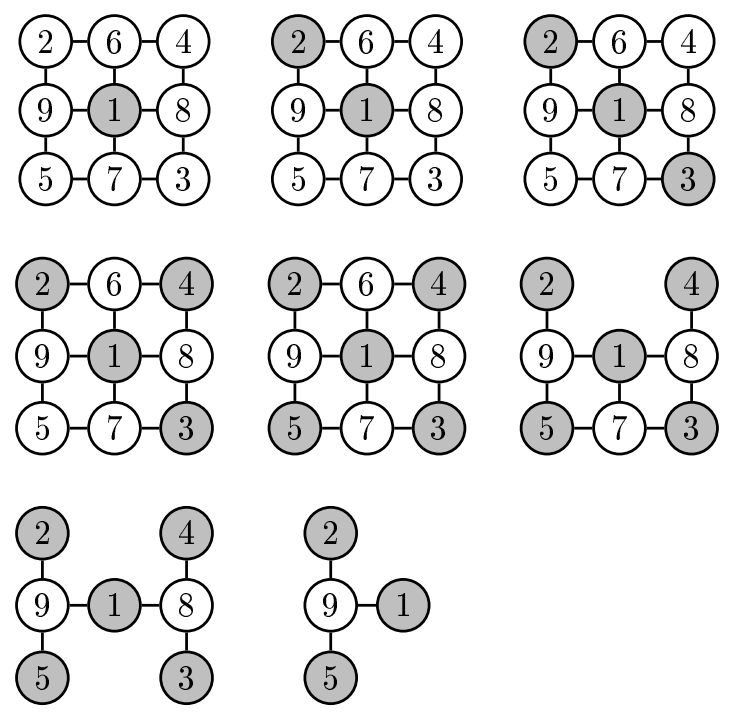

$$
F_{\max }=5
$$

Figure 4: The value of $F_{\max }$ is a consequence of the initial vertex numbering. 
Table 2: Comparison of different vertex linear orderings

\begin{tabular}{|c|c|c|c|c|c|c|c|}
\hline Problem & $F_{\max }\left(\mathcal{N}_{0}\right)$ & $F_{\max }\left(\mathcal{N}_{1}\right)$ & $F_{\max }\left(\mathcal{N}_{2}\right)$ & Problem & $F_{\max }\left(\mathcal{N}_{0}\right)$ & $F_{\max }\left(\mathcal{N}_{1}\right)$ & $F_{\max }\left(\mathcal{N}_{2}\right)$ \\
\hline $\mathrm{G}(30, .1)$ & 12.4 & 9.6 & 8.4 & $\mathrm{G}(40, .5)$ & 34.0 & 34.4 & 33.6 \\
\hline $\mathrm{G}(40, .1)$ & 20.8 & 16.4 & 15.0 & $\mathrm{G}(50, .5)$ & 43.4 & 43.4 & 41.8 \\
\hline $\mathrm{G}(50, .1)$ & 29.4 & 26.2 & 22.0 & $\mathrm{G}(60, .5)$ & 53.8 & 53.8 & 52.4 \\
\hline $\mathrm{G}(60, .1)$ & 36.8 & 32.8 & 28.0 & $\mathrm{G}(30, .9)$ & 27.8 & 27.8 & 27.2 \\
\hline $\mathrm{G}(70, .1)$ & 44.0 & 43.0 & 34.2 & $\mathrm{G}(40, .9)$ & 37.8 & 37.8 & 36.4 \\
\hline $\mathrm{G}(80, .1)$ & 55.0 & 50.8 & 42.8 & $\mathrm{G}(50, .9)$ & 47.4 & 47.4 & 46.8 \\
\hline $\mathrm{G}(90, .1)$ & 63.6 & 60.0 & 51.8 & $\mathrm{G}(60, .9)$ & 57.8 & 57.8 & 57.0 \\
\hline $\mathrm{G}(30, .5)$ & 24.8 & 24.6 & 22.6 & $\mathrm{G}(70, .9)$ & 67.6 & 67.4 & 67.0 \\
\hline mug88_1 & 20 & 13 & 8 & mug88_25 & 20 & 13 & 8 \\
\hline mug100_1 & 17 & 22 & 7 & mug100_25 & 19 & 16 & 8 \\
\hline 1-FullIns4 & 61 & 71 & 46 & 1-FullIns5 & 187 & 216 & 132 \\
\hline 2-FullIns3 & 37 & 28 & 22 & 2-FullIns4 & 157 & 105 & 93 \\
\hline 2-FullIns5 & 637 & 417 & 359 & 3-FullIns3 & 61 & 45 & 36 \\
\hline 3-FullIns4 & 321 & 247 & 200 & 4-FullIns3 & 91 & 57 & 49 \\
\hline 4-FullIns4 & 571 & 332 & 302 & 5-FullIns3 & 127 & 127 & 64 \\
\hline 5-FullIns4 & 925 & 772 & 447 & 1-Inser_4 & 31 & 40 & 32 \\
\hline 1-Inser_5 & 98 & 145 & 91 & 1-Inser_6 & 300 & 406 & 276 \\
\hline 2-Inser_4 & 48 & 56 & 52 & 2-Inser_5 & 197 & 251 & 240 \\
\hline 3-Inser_3 & 13 & 13 & 16 & 3-Inser_4 & 69 & 92 & 84 \\
\hline 3-Inser_5 & 350 & 578 & 436 & 4-Inser_3 & 15 & 15 & 20 \\
\hline 4-Inser_4 & 94 & 120 & 124 & miles 250 & 67 & 48 & 16 \\
\hline le450_5a & 381 & 368 & 339 & le450_5b & 386 & 382 & 343 \\
\hline le450_5c & 397 & 401 & 385 & le450_5d & 400 & 403 & 385 \\
\hline le450_15a & 373 & 365 & 339 & le450_15b & 378 & 372 & 336 \\
\hline le450_15c & 420 & 418 & 396 & le450_15d & 416 & 415 & 401 \\
\hline le450_25a & 351 & 348 & 293 & le450_25c & 416 & 412 & 394 \\
\hline le450_25d & 411 & 409 & 384 & & & & \\
\hline
\end{tabular}


vertex with too few neighbors in the boundary set. To avoid this case we add a reduction on the graph before each k-coloring.

\section{7 $\quad$ Vertex reduction}

Before each k-coloring, we delete some vertices of the graph by using the following property: for each vertex $x$, if the degree of $x$ is strictly lower than $k, x$ and its adjacent edges can be erased from the graph [13]. Indeed, assume $x$ has $k-1$ neighbors. In the worst case, those neighbors must have different colors. Then the vertex $x$ can take the $k^{\text {th }}$ color. It does not interfere in the coloring of the remaining vertices because all its neighbors have already been colored. Therefore we can consider from the beginning that it will take a color unused by its neighbors and delete it from the graph before the coloring. We apply this principle recursively by examining the remaining vertices until having totally reduced the graph or being enable to delete any other vertex.

\section{Experimental results}

Our $L D C$ algorithms have been implemented on a PC AMD Athlon Xp 2000+ in $\mathrm{C}$ language. We performed tests on random graphs and on benchmark instances used at the computational symposium COLOR02.

\subsection{Random graphs}

Random graphs $G_{N, d}$ have been created. For each graph, $N$ is the number of vertices and $d$ its density. We generated graphs with densities $0.1,0.5$ and 0.9 . We give the results of our experiments over average of 5 instances in Table 3. For each type of graph, we give the number of edges $M$ and the values $L B$ and $U B$ of our initial bounds. Algorithms Kdic $-L D C$ and Kseq $-L D C$ have been applied to each instance, using a CPU time limit of half an hour. For each algorithm, we give the average chromatic number and the CPU time when the program is able to compute. Otherwise, when less than 5 instances have been solved, we indicate the number of solved instances in brackets. Starting from $N=30$, we increase the size of random graphs by step 10 until less than 3 instances are solved.

Our algorithms are not very efficient on random graphs in comparison with the results of other exact methods [21, 15, 7]. Indeed, Desrosiers, Galinier and Hertz are able to solve graphs with density 0.5 and with up to 100 vertices. This is mainly due to the fact that edges repartition in the graph is homogeneous, 
Table 3: Results on random graphs

\begin{tabular}{|c|c|c|c|c|c|c|c|}
\hline & & & & \multicolumn{4}{|c|}{ LDC } \\
\cline { 5 - 8 } Problem & $M$ & LB & UB & Kdic & T & Kseq & T \\
\hline \hline $\mathrm{G}(30, .1)$ & 43.8 & 2.8 & 3.0 & 3.0 & 0.00 & 3.0 & 0.00 \\
$\mathrm{G}(40, .1)$ & 75.8 & 3.0 & 3.6 & 3.2 & 0.00 & 3.2 & 0.00 \\
$\mathrm{G}(50, .1)$ & 123.2 & 3.0 & 4.2 & 4.0 & 0.00 & 4.0 & 0.78 \\
$\mathrm{G}(60, .1)$ & 177.0 & 3.0 & 4.6 & $4.0[2]$ & 0.00 & 4.0 & 2.54 \\
$\mathrm{G}(70, .1)$ & 237.8 & 3.0 & 5.0 & & & 4.0 & 2.66 \\
$\mathrm{G}(80, .1)$ & 320.4 & 3.0 & 5.0 & & & $4.2[4]$ & 103.16 \\
$\mathrm{G}(90, .1)$ & 397.0 & 3.2 & 5.6 & & & $4.6[3]$ & 104.80 \\
$\mathrm{G}(100, .1)$ & 477.2 & 3.0 & 5.6 & & & $5.0[4]$ & 190.99 \\
$\mathrm{G}(110, .1)$ & 604.4 & 3.8 & 6.0 & & & $5.0[4]$ & 91.90 \\
$\mathrm{G}(120, .1)$ & 711.8 & 3.2 & 6.4 & & & {$[0]$} & $*$ \\
\hline $\mathrm{G}(30, .5)$ & 211.2 & 5.2 & 8.2 & 7.0 & 11.18 & 7.0 & 2.92 \\
$\mathrm{G}(40, .5)$ & 380.0 & 6.2 & 9.8 & 8.2 & 6.19 & 8.2 & 6.16 \\
$\mathrm{G}(50, .5)$ & 608.6 & 6.2 & 12.0 & $6.0[3]$ & 97.93 & $9.4[3]$ & 83.12 \\
$\mathrm{G}(60, .5)$ & 881.8 & 6.6 & 12.8 & {$[0]$} & $*$ & {$[0]$} & $*$ \\
\hline $\mathrm{G}(30, .9)$ & 392 & 15.2 & 16.2 & 15.8 & 0.02 & 15.8 & 0.01 \\
$\mathrm{G}(40, .9)$ & 699.8 & 18.2 & 22.6 & 18.8 & 7.11 & 18.8 & 0.44 \\
$\mathrm{G}(50, .9)$ & 1103.6 & 20.8 & 25.4 & 22.8 & 13.2 & 22.8 & 10.79 \\
$\mathrm{G}(60, .9)$ & 1606.6 & 24.0 & 30.6 & {$[0]$} & $*$ & $26.2[4]$ & 105.02 \\
$\mathrm{G}(70, .9)$ & 2172.6 & 25.0 & 33.4 & & & $28[1]$ & 684.8 \\
\hline
\end{tabular}


inducing a large linearwidth. For a graph with 50 vertices, we have (see Table 2) $F_{\text {max }}\left(\mathcal{N}_{2}\right)=41$ when the graph density is 0.5 and $F_{\max }\left(\mathcal{N}_{2}\right)=46$ when the graph density is 0.9 . Nevertheless, results of Table 3 reflect clearly the possibilities and the limits of our method. We are able to solve graphs with density 0.9 and 60 vertices. These graphs have a very large linearwidth but are also very constrained : at each step only few configurations are generated despite the big size of the boundary sets. The decomposition method is not efficient on random graphs with density 0.5 . Indeed, the large size of the linearwidth induces the possibility to generate an exponential number of configurations and the 0.5 density does not limit enough the number of valid configurations. Our linear-decomposition method works better on graphs with small density. Indeed, the maximum size of the boundary set can be very reduced by the vertex numbering $\mathcal{N}_{2}$. For this kind of random graphs, we are able to solve instances with up to 100 vertices.

\subsection{DIMACS and COLOR02 instances}

We performed tests on benchmark instances used at the computational symposium COLOR02, among which well-known DIMACS instances (see description of the instances at http://mat.gsia.cmu.edu/COLOR02).

We first apply on each instance the lower bound and upper bound described in section 3.5. Results are reported in table 4. For each graph we give the number of vertices $N$, the number of edges $M$, and the values $L B$ and $U B$ of our initial bounds. At this stage, the lower bound equals the upper bound for some of the DIMACS instances, so we did not apply any k-coloring algorithm on these graphs.

We compare our $L D C$ algorithms with the recent results of the exact Branchand-Cut algorithm of Mendez Diaz and Zabala [9]. We use a CPU time limit of half an hour. The results of our experiments are reported in table 5. The time column for $\mathrm{B} \& \mathrm{C}$ is only indicative since the results are from different machines. They used $\mathrm{C}++$ code using ABACUS framework and CPLEX 6.0 LP solver on a Sun ULTRA workstation. Their CPU time limit was two hours. Comparing their time results for the same implementation of Dsat that we use, our computer seems to run three times faster than their computer. We report also the results of Desrosiers, Galinier and Hertz [7] in VCS columns. Column btk contains the number of backtracks needed by their exact algorithm to solve an instance reduced to a vertex-critical subgraph. Values are enclosed in parentheses when their algorithm is not able to find the chromatic number 
Table 4: Lower bound and Upper bound results

\begin{tabular}{|c|c|c|c|c|c|c|c|c|c|}
\hline Problem & $\mathrm{N}$ & M & LB & UB & Problem & $\mathrm{N}$ & M & LB & UB \\
\hline fpsol2.i.1 & 496 & 11654 & 65 & 65 & 1-FullIns3 & 30 & 100 & 3 & 5 \\
\hline fpsol2.i.2 & 451 & 8691 & 30 & 30 & 1-FullIns4 & 93 & 593 & 3 & 6 \\
\hline fpsol2.i.3 & 425 & 8688 & 30 & 30 & 1-FullIns5 & 282 & 3247 & 3 & 8 \\
\hline inithx.i.1 & 864 & 18707 & 54 & 54 & 2-FullIns3 & 52 & 201 & 4 & 5 \\
\hline inithx.i.2 & 645 & 13979 & 31 & 31 & 2-FullIns4 & 212 & 1621 & 4 & 6 \\
\hline inithx.i.3 & 621 & 13969 & 31 & 31 & 2-FullIns5 & 852 & 12201 & 4 & 7 \\
\hline le450-5a & 450 & 5714 & 5 & 11 & 3-FullIns3 & 80 & 346 & 5 & 6 \\
\hline le450-5b & 450 & 5734 & 5 & 11 & 3-FullIns4 & 405 & 3524 & 2 & 7 \\
\hline le $450-5 \mathrm{c}$ & 450 & 9803 & 4 & 13 & 4-FullIns3 & 114 & 541 & 6 & 7 \\
\hline le450-5d & 450 & 9757 & 4 & 12 & 4-FullIns4 & 690 & 6650 & 2 & 8 \\
\hline le450-15a & 450 & 8168 & 13 & 18 & 5-FullIns3 & 154 & 792 & 7 & 8 \\
\hline le450-15b & 450 & 8169 & 15 & 17 & 5-FullIns4 & 1085 & 11395 & 2 & 9 \\
\hline le450-15c & 450 & 16680 & 11 & 25 & 1-Inser_4 & 67 & 232 & 2 & 5 \\
\hline le450-15d & 450 & 16750 & 11 & 26 & 1-Inser_5 & 202 & 1227 & 2 & 6 \\
\hline le450-25a & 450 & 8260 & 20 & 25 & 1-Inser_6 & 607 & 6337 & 2 & 7 \\
\hline le450_25b & 450 & 8263 & 25 & 25 & 2-Inser_3 & 37 & 72 & 2 & 4 \\
\hline le450-25c & 450 & 17343 & 22 & 30 & 2-Inser_4 & 149 & 541 & 2 & 5 \\
\hline le450-25d & 450 & 17425 & 19 & 29 & 2-Inser_5 & 597 & 3936 & 2 & 6 \\
\hline mulsol.i.1 & 197 & 3925 & 49 & 49 & 3-Inser_3 & 56 & 110 & 2 & 4 \\
\hline mulsol.i.2 & 188 & 3885 & 31 & 31 & 3-Inser_4 & 281 & 1046 & 2 & 5 \\
\hline mulsol.i.3 & 184 & 3916 & 31 & 31 & 3-Inser_5 & 1406 & 9695 & 2 & 6 \\
\hline mulsol.i.4 & 185 & 3946 & 31 & 31 & 4-Inser_3 & 79 & 156 & 2 & 4 \\
\hline mulsol.i.5 & 186 & 3973 & 31 & 31 & 4-Inser_4 & 475 & 1795 & 2 & 5 \\
\hline school1 & 385 & 19095 & 14 & 14 & mug100-1 & 100 & 166 & 3 & 4 \\
\hline school1_nsh & 352 & 14612 & 14 & 14 & mug100-25 & 100 & 166 & 3 & 4 \\
\hline zeroin.i.1 & 211 & 4100 & 49 & 49 & mug88-1 & 88 & 146 & 3 & 4 \\
\hline zeroin.i.2 & 211 & 3541 & 30 & 30 & mug88-25 & 88 & 146 & 3 & 4 \\
\hline zeroin.i.3 & 206 & 3540 & 30 & 30 & games120 & 120 & 638 & 9 & 9 \\
\hline anna & 138 & 493 & 11 & 11 & miles 250 & 128 & 387 & 7 & 8 \\
\hline david & 87 & 812 & 11 & 11 & miles 500 & 128 & 2340 & 20 & 20 \\
\hline homer & 561 & 1629 & 13 & 13 & miles 750 & 128 & 4226 & 31 & 31 \\
\hline huck & 74 & 602 & 11 & 11 & miles1000 & 128 & 6432 & 42 & 42 \\
\hline jean & 80 & 508 & 10 & 10 & miles1500 & 128 & 10396 & 73 & 73 \\
\hline
\end{tabular}


within the time limit.

The $F_{\max }$ column indicates for each graph the maximal value of the boundary set reached by our algorithm. We give the chromatic number of the graph and the CPU time when the program is able to compute. Otherwise, asterisks in the time column indicate that the program exceeded the time limit. In this case, we report the best lower and upper bounds of the chromatic number obtained by the algorithm.

The results of Kseq- $L D C$ are equivalent or better than those of Kdic- $L D C$ on the tested instances, that is why we report only Kseq- $L D C$ results. Our algorithm runs very fast on mug instances ( 0.0 or 0.1 second), because the vertex linear ordering chosen provides small linearwidths for these graphs $\left(F_{\max }\right.$ column). For these instances, the results of $K$ dic- $L D C$ and $K$ seq- $L D C$ are the same, since $U B=L B+1$. B\&C and $V C S$ found the chromatic number, but spent time to explore the Branch-and-Cut tree. Our algorithms are also able to solve some instances which have a large linearwidth, because all the partitions of the boundary set have not necessarily to be generated. We found the chromatic number of 13 instances, 12 of which solved in less than 30 seconds. The $L D C$ algorithm is the first to our knowledge to find the chromatic number of the instance 4-Inser_3 (bolded in table 5) with an exact method.

\section{Conclusions}

In this paper, we have presented an original method to solve the graph coloring problem by an exact way. This method has the advantage of solving easily large instances which have a small linearwidth, such as mug instances, and allows us to solve the difficult instance 4-Inser_3. We consider using the linear-decomposition mixed with heuristics approach to deal with unbounded linearwidth instances.

\section{References}

[1] H. L. Bodlaender. A tourist guide through treewidth. Acta Cybernetica, 11:1-21, 1993.

[2] H. L. Bodlaender. A linear time algorithm for finding tree-decompositions of small treewidth. SIAM Journal on Computing, 25(6):1305-1317, 1996.

[3] D. Brelaz. New methods to color the vertices of a graph. Communications of the ACM, 22(4):251-256, april 1979. 
Table 5: Results on COLOR02 instances

\begin{tabular}{|c|c|c|c|c|c|c|c|}
\hline \multirow[b]{2}{*}{ Problem } & \multicolumn{3}{|c|}{ LDC } & \multicolumn{2}{|c|}{$\mathrm{B} \& \mathrm{C}$} & \multicolumn{2}{|r|}{ VCS } \\
\hline & $F_{\max }$ & $k$ & $\mathrm{~T}$ & $k$ & $\mathrm{~T}$ & $k$ & $b t k$ \\
\hline mug88_1 & 8 & 4 & 0.0 & 4 & 485 & 4 & 2204467 \\
\hline mug88_25 & 8 & 4 & 0.0 & 4 & 1690 & 4 & 942961 \\
\hline mug100_1 & 7 & 4 & 0.1 & 4 & 4029 & 4 & 1406570 \\
\hline mug100_25 & 8 & 4 & 0.0 & 4 & 5498 & 4 & 974170 \\
\hline 1-FullIns4 & 27 & 5 & 7.3 & 5 & 703 & 5 & 6 \\
\hline 1-FullIns5 & 26 & $4-6$ & $*$ & $4-6$ & $*$ & 6 & 271 \\
\hline 2-FullIns3 & 9 & 5 & 0.0 & 5 & 3 & 5 & 1 \\
\hline 2-FullIns4 & 19 & $5-6$ & $*$ & $5-6$ & $*$ & 6 & 8 \\
\hline 2-FullIns5 & 20 & $5-7$ & * & $5-7$ & $*$ & 7 & 715 \\
\hline 3-FullIns3 & 11 & 6 & 0.0 & 6 & 1 & 6 & 0 \\
\hline 3-FullIns4 & 20 & $3-7$ & $*$ & $6-7$ & $*$ & 7 & 10 \\
\hline 4-FullIns3 & 11 & 7 & 0.0 & 7 & 3 & 7 & 1 \\
\hline 4-FullIns4 & 17 & $4-8$ & $*$ & $7-8$ & $*$ & 8 & 12 \\
\hline 5-FullIns3 & 13 & 8 & 0.0 & 8 & 3 & 8 & 1 \\
\hline 5-FullIns4 & 17 & $3-9$ & $*$ & $7-9$ & $*$ & & \\
\hline 1-Inser_4 & 21 & $4-5$ & $*$ & $2-5$ & $*$ & 5 & 104296036 \\
\hline 1-Inser_5 & 20 & $3-6$ & * & $4-6$ & $*$ & $*$ & (133727661) \\
\hline 1-Inser_6 & 18 & $3-7$ & * & $4-7$ & $*$ & $*$ & $(50929137)$ \\
\hline 2-Inser_4 & 28 & $3-5$ & $*$ & $4-5$ & $*$ & $*$ & $(154902785)$ \\
\hline 2-Inser_5 & 18 & $3-6$ & $*$ & $3-6$ & $*$ & $*$ & $(48458541)$ \\
\hline 3-Inser_3 & 16 & 4 & 23.3 & 4 & 10 & 4 & 723616 \\
\hline 3-Inser_4 & 28 & $3-5$ & $*$ & $3-5$ & $*$ & $*$ & $(95076991)$ \\
\hline 3-Inser_5 & 17 & $3-6$ & $*$ & $3-6$ & $*$ & $*$ & $(13784327)$ \\
\hline 4-Inser_3 & 20 & 4 & 1774 & $3-4$ & $*$ & $*$ & $(228367528)$ \\
\hline 4-Inser_4 & 27 & $3-5$ & * & $3-5$ & $*$ & $*$ & $(70891706)$ \\
\hline miles 250 & 9 & 8 & 0.0 & & & 8 & 0 \\
\hline le450_5a & 15 & $5-9$ & $*$ & $5-9$ & $*$ & 5 & 0 \\
\hline le450_5b & 16 & $5-9$ & $*$ & $5-9$ & $*$ & 5 & 0 \\
\hline le450_5c & 22 & $5-6$ & $*$ & & & 5 & 0 \\
\hline le450_5d & 46 & $5-7$ & $*$ & $5-10$ & $*$ & 5 & 0 \\
\hline le450_15a & 21 & $15-18$ & $*$ & $15-17$ & $*$ & 15 & 0 \\
\hline le450_15b & 21 & $15-17$ & * & $15-17$ & $*$ & 15 & 0 \\
\hline le450_15c & 21 & $15-25$ & $*$ & $15-24$ & $*$ & 15 & 0 \\
\hline le450_15d & 21 & $15-26$ & $*$ & $15-23$ & * & 15 & 0 \\
\hline le450_25a & 25 & 25 & 0.0 & & & 25 & 0 \\
\hline le450_25c & 30 & $25-28$ & $*$ & $25-28$ & $*$ & 25 & 0 \\
\hline le450_25d & 30 & $25-28$ & $*$ & $225-28$ & $*$ & 25 & 0 \\
\hline
\end{tabular}


[4] J. Carlier and C. Lucet. A decomposition algorithm for network reliability evaluation. Discrete Appl. Math., 65:141-156, 1996.

[5] D. de Werra. An introduction to timetabling. European Journal of Operation Research, 19:151-162, 1985.

[6] D. de Werra. On a multiconstrained model for chromatic scheduling. Discrete Appl. Math., 94:171-180, 1999.

[7] C. Desrosiers, P. Galinier, and A. Hertz. Efficient algorithms for finding critical subgraphs. Cahiers du GERAD, G-2004-31, 2004.

[8] I. Mendez Diaz and P. Zabala. A branch-and-cut algorithm for graph coloring. In Computational Symposium on Graph Coloring and its Generalizations (COLOR02), Ithaca, N-Y, september 2002.

[9] I. Mendez Diaz and P. Zabala. A branch-and-cut algorithm for graph coloring. Optimization Online: Combinatorial Optimization, september 2003.

[10] N. Funabiki and T. Higashino. A minimal-state processing search algorithm for graph coloring problems. IEICE Transactions on Fundamentals, E83-A(7):14201430, 2000.

[11] P. Galinier and J.K. Hao. Hybrid evolutionary algorithms for graph coloring. Journal of Combinatorial Optimization, 3(4):379-397, 1999.

[12] M. R. Garey and D. S. Johnson. Computers and Intractability - A Guide to the Theory of NP-Completeness. Freeman, San Francisco, 1979.

[13] F. Glover, M. Parker, and J. Ryan. Coloring by tabu branch and bound. In Trick and Johnson [28], pages 285-308.

[14] M. C. Golumbic. Algorithmic Graph Theory and Perfect Graphs. Academic Press, New York, 1980.

[15] F. Herrmann and A. Hertz. Finding the chromatic number by means of critical graphs. ACM Journal of Experimental Algorithmics, 7(10):1-9, 2002.

[16] A. Hertz and D. De Werra. Using tabu search techniques for graph coloring. Computing, 39:345-351, 1987.

[17] M. Kubale and B. Jackowski. A generalized implicit enumeration algorithm for graph coloring. Communications of the ACM, 28(4):412-418, 1985.

[18] C. Lucet. Méthode de décomposition pour l'évaluation de la fiabilité des réseaux. PhD thesis, Université de Technologie de Compiègne, 1993.

[19] J.F. Manouvrier. Méthode de décomposition pour résoudre des problèmes combinatoires sur les graphes. PhD thesis, Université de Technologie de Compiègne, 1998.

[20] B. Manvel. Extremely greedy coloring algorithms. In F. Harary and J.S. Maybee, editors, Graphs and applications: Proceedings of the First Colorado Symposium on Graph Theory, pages 257-270, New York, 1985. John Wiley \& Sons. 
[21] A. Mehrotra and M. A. Trick. A column generation approach for graph coloring. INFORMS Journal on Computing, 8(4):344-354, 1996.

[22] C. A. Morgenstern. Distributed coloration neighborhood search. In Trick and Johnson [28], pages 335-357.

[23] N. Robertson and P. Seymour. Graph minors. ii. algorithmic aspects of tree-width. Journal of Algorithms, 7:309-322, 1986.

[24] D. G. Corneil S. Arnborg and A. Proskurowski. Complexity of finding embeddings in a k-tree. SIAM Journal on Algebraic and Discrete Methods, 8:277-284, 1987.

[25] T. J. Sager and S. Lin. A pruning procedure for exact graph coloring. ORSA Journal on Computing, 3:226-230, 1991.

[26] S. Sen Sarma and S. K. Bandyopadhyay. Some sequential graph colouring algorithms. International Journal of Electronic, 67(2):187-199, 1989.

[27] E. Sewell. An improved algorithm for exact graph coloring. In Trick and Johnson [28], pages 359-373.

[28] Michael A. Trick and David S. Johnson, editors. Cliques, Coloring, and Satisfiability: Proceedings of the Second DIMACS Implementation Challenge. American Mathematical Society, 1993. 